\title{
Erradicación de diabetes en Guatemala: Un sueño posible
}

\author{
José Antonio Cornejo Guerra \\ Departamento de Medicina Interna, Hospital General San Juan de Dios y \\ Facultad de Ciencias Médicas, Universidad de San Carlos de Guatemala, Guatemala.
}

*Autor al que se dirige la correspondencia: jacornejoguerra@gmail.com

Recibido: 25 de febrero 2015 / Revisión: 24 de abril de 2015 / Aceptado: 04 de mayo 2015

\section{Resumen}

\begin{abstract}
T a diabetes mellitus es un problema de salud con alcances económicos y sociales. La prevalencia mundial está Laumentando: se estima que para 1985 había 30 millones de personas con diabetes, 177 millones en el año 2000 y se estima que para el año 2030 habrá 438 millones de diabéticos. En Guatemala el 8\% de la población tiene diabetes y se desconoce el porcentaje no diagnosticado y en pre-diabetes; además el país tiene índices de obesidad, sobrepeso y sedentarismo altos, situación que predispone a la aparición de la enfermedad. En el mundo cada 6 segundos muere alguien a causa de complicaciones relacionadas con diabetes y en Guatemala ocupa la tercera causa de mortalidad general. Existen varios tipos de diabetes, sin embargo, el ensayo se enfoca en la diabetes tipo 2 debido a que representa el $90 \%$ de los casos pero sobre todo por que es prevenible. Para el autor existen tres momentos importantes de los pacientes diabéticos: (1) paciente sano y con riesgo qué enfermará de diabetes, (2) paciente diabético que tendrá complicaciones y (3) pacientes con complicaciones que morirán. En los tres momentos se puede intervenir directamente, sin embargo, existen deficiencias como la ausencia de programas de prevención y la ineficiencia del sistema asistencial público. El autor propone posibles soluciones en base a evidencia científica para detener el aumento de casos de diabetes mellitus, disminuir las complicaciones micro y macro vasculares en los pacientes y principalmente, generar una cultura preventiva en Guatemala.
\end{abstract}

\section{Abstract}

$\mathrm{D}$ abetes is a health problem with social and economic consequences. The worldwide prevalence of diabetes is raising; by 1985 there were 30 million of people with diabetes, 177 million by the year 2000 and it is estimated that in 2030 there will be around 438 million people with diabetes. The prevalence of diabetes in Guatemala it's around $8 \%$ of the population, however it is unknown the prevalence of the underdiagnosed and pre-diabetes. Guatemala has high obesity, overweight and sedentary index which increase the risk of diabetes. Worldwide every 6 seconds somebody dies as a consequence of diabetes and in Guatemala represents the third cause in general mortality. The analysis is made about type 2 diabetes which represents $90 \%$ of all diabetes and in general is preventable. The author proposes 3 moments in the diabetes natural history: (1) healthy patients that will become diabetics, (2) diabetics who will have a complication related to the disease and (3) complicated patients who will decease. During these three moments there are interventions to be done, however there are non-preventable programs and the inefficiency of the public assistant system. The author proposes possible solutions in base of scientific evidence to stop the raising of diabetes, lowering micro and macro vascular complications and overall to generate a preventive culture in Guatemala. 


\section{Introducción}

El autor se declara un experto en diabetes, mas no por el tema académico, sino por los enfermos que ha visto enterrar. La diabetes más allá de ser un problema de salud, se torna en una problemática de magnitud social y económica. El presente ensayo aborda la enfermedad desde su prevalencia regional y las proyecciones estimadas; la carga económica que representa para los sistemas de salud, lo que el Ministerio de Salud Pública y Asistencia Social (MSPAS) actualmente está ejecutando para el control de la diabetes y las perspectivas que el autor considera que pueden corregir el curso actual. En base a evidencia científica, se demostrará el por qué Guatemala es un país en riesgo para el aumento en incidencia de diabetes, así como las dimensiones reales del problema, por ejemplo, según la Federación Internacional de Diabetes (FID) cada seis segundos muere una persona con diabetes en el mundo (2013).

\section{Inicio}

Prevalencia según lo expresado por la Real Academia Española (2014) denota, la acción y efecto de prevalecer, del latín praevalescěre, del verbo intransitivo perdurar o subsistir. En epidemiología prevalence prevalecia en español-, es el número total de casos de una enfermedad dada en una población especifica en un tiempo determinado (Wiley Online Library. Cochrane Library, 2015).

Sin embargo, para fines del presente ensayo se pretende que el lector comprenda el poderío de la diabetes para persistir entre la humanidad, a pesar de los miles de esfuerzos que se llevan a cabo para su control, y más fuerte aún es la capacidad para continuar creciendo.

La prevalencia mundial de diabetes mellitus se ha incrementado de manera importante, para el año 1985 se estimaron 30 millones de casos y para el 2000 aumentó alrededor de 177 millones de diabéticos (World Heart Organization, 2015), lo cual significa que se multiplicó por más de cinco veces. Basados en los datos de la FID para el 2030 se calcula habrá 438 millones de individuos con diabetes (2013).

En cuanto a Latinoamérica, se estima que la prevalencia de diabetes es de 24.1 millones de personas o el $8 \%$ de la población adulta. Esta cifra se espera que aumente $60 \%$ para el 2035 . Además, se sabe que otros 22.4 millones de personas que representan el $7.4 \%$ de la población adulta tienen tolerancia anormal de la glucosa (FID, 2013) que es un estado de pre-diabetes, lo que significa que en un momento dado algún porcentaje de estos 22 millones de personas pueden tornarse diabéticos si no modifican su estilo de vida, que incluye actividad física, dieta balanceada y tratamiento farmacológico adecuado. De los países de la región con mayor prevalencia se menciona a Puerto Rico (13\%) de su población total, seguido por Nicaragua (12.4\%), República Dominicana (11.3\%) y $(10.9 \%)$ para Guatemala (FID, 2013). Aunque según el MSPAS (2012) la prevalencia de diabetes es de $8 \%$ de la población. Existe un dato importante de mencionar y es que es conocido que el impacto de la diabetes es principalmente urbano, se dice que en las comunidades rurales es un problema importante; ilustrado por la FID (2013) que asegura que "los pueblos indígenas son especialmente vulnerables a la diabetes". Según los datos proporcionados por el Instituto Nacional de Estadística (INE), aproximadamente el $40 \%$ de la población se compone de pueblos indígenas (2013a).

En cuanto a la mortalidad, en Guatemala para el año 2012 la diabetes mellitus representó la tercera causa de muerte (11.9\%) en la población general, la segunda fue infarto agudo al miocardio (16.1\%) y la primera fue neumonía (21.7\%). Paradójicamente, la diabetes puede predisponer ambas es un factor de riesgo para infarto agudo al miocardio y neumonía (INE, 2013a). Con las cifras anteriores se pretende que el lector conozca el panorama general actual de la diabetes y las proyecciones para el futuro, además de entender que no es un problema ajeno, es decir: nos enfermamos de diabetes y morimos de ella.

Como se mencionó antes, es importante comprender el gasto que la diabetes genera. Comparada con los países centroamericanos, en el 2003, Guatemala tuvo los costos directos e indirectos más altos de la región: USD 291 millones y USD 549 millones respectivamente; y en contraste, el tercer menor gasto per-cápita en salud del istmo centroamericano (MSPAS, 2008). En el dato anterior es importante recalcar que los gastos indirectos superan a los gastos directos, esto quizá, por la falta de acceso a sistemas de salud, es decir que los costos generados por ausentismo laboral por enfermedad, incapacidad, jubilación prematura, mortalidad prematura y absentismo laboral de quienes cuidan a personas con diabetes (Rhys, 2002), son mayores que los costos efectivos en atención de la enfermedad.

Para los sistemas de salud la diabetes representa una fuerte carga, por ejemplo afrontar el gasto sanitario y la carga económica de la enfermedad; el gasto sanita- 
rio se refiere a lo que los sistemas de salud y las personas con diabetes gastan en la prevención, tratamiento y tratamiento de complicaciones y la carga económica se refiere a lo que los pacientes gastan en su enfermedad. Se estima que en 2013 se gastó un promedio de USD 1,437 por persona con diabetes a nivel mundial en el tratamiento y control de la enfermedad; de esta cantidad únicamente el $20 \%$ se utilizó en países de ingresos medio y bajos, donde vive el $80 \%$ de las personas con diabetes (FID, 2013). Lo que significa que en los países más pobres, donde paradójicamente viven la mayoría de diabéticos, se cuenta con al menos un quinto del gasto mundial para la enfermedad; fórmula que no termina de tener lógica.

Por ejemplo, el gasto sanitario anual estimado debido a la diabetes fue USD 5,621 por persona con diabetes en los países de ingresos altos, en comparación con USD 365 en países de ingreso medio y bajos (FID, 2013) como Guatemala. En América Latina se estima que las familias pagan entre el 40 y $60 \%$ de los gastos médicos de su propio bolsillo (Barceló, Aedo, Rajpathak, $\&$ Robles, 2003). Según el INE se estima que el 53.7\% de la población guatemalteca está en pobreza general y un $13.3 \%$ en pobreza extrema (2013b); por lo que se puede inferir que para más de la mitad de la población es económicamente imposible costearse el tratamiento de diabetes y el sistema de salud pública no provee un tratamiento adecuado, afirmación que será respaldada más adelante.

Según la visión del autor, existen tres momentos en la diabetes mellitus: (1) las personas sanas con factores de riesgo que enfermarán de diabetes mellitus; (2) las personas enfermas de diabetes mellitus que tendrán complicaciones relacionadas con la enfermedad; y (3) las personas con diabetes mellitus y complicaciones a causa de la enfermedad que finalmente morirán. Se abordará la problemática de cada grupo procurando responder las siguientes interrogantes, ¿qué se está haciendo actualmente en Guatemala? Si existieran medidas, ¿están siendo eficientes? Y por último, se plantearán posibles soluciones.

$\mathrm{Al}$ analizar a las personas con factores de riesgo que enfermarán de diabetes se debe iniciar una breve explicación sobre el proceso que lleva a los pacientes a tornarse diabéticos tipo 2, ya que la enfermedad no tiene una etiología aguda, sino es un proceso lento. El aumento en la diabetes tipo 2 se relaciona con cambios del estilo de vida como la disminución del nivel de actividad física y condiciones como el sobrepeso y la obesidad. Estos cambios ambientales aunados a predis- posición genética, aumentan la resistencia a la insulina, la cual, se asocia a falla progresiva de las células $\beta$ pancreáticas, resultando en aumento de la glicemia en rangos no diabéticos. Además del riesgo para desarrollar diabetes en estos pacientes, la resistencia a insulina y anormalidad en la secreción de insulina se acompañan por otros factores de riesgo cardiovasculares de los individuos como hipertensión arterial y dislipidemia (Nathan et al., 2007). ¡Ojo! que durante el proceso antes descrito los síntomas de los pacientes pueden ser leves o incluso, estar ausentes, por lo cual este proceso es raramente detectado a excepción de exámenes de rutina, de los cuales los pacientes jóvenes serán los más vulnerables, por la creencia que esta enfermedad es de adultos mayores.

La reducción posterior de la secreción de la insulina resulta en aumento de la glicemia y el desarrollo de diabetes. La transición de anormalidades metabólicas tempranas que preceden la diabetes son: la glucosa anormal en ayuno y tolerancia anormal de la glucosa. Actualmente se estima que la mayoría de individuos (alrededor de 70\%) en estos estados pre-diabéticos desarrollarán diabetes (Nathan et al., 2007). Para aclarar los conceptos, la "glucosa anormal en ayuno" se define como una glucosa plasmática en ayunas $\geq 100 \mathrm{mg} / \mathrm{dl}$ $\mathrm{y}<126 \mathrm{mg} / \mathrm{dl}$, mientras que la "tolerancia anormal de la glucosa" se define como la concentración plasmática de glucosa $\geq 140 \mathrm{mg} / \mathrm{dl} \mathrm{y}<200 \mathrm{mg} / \mathrm{dl}$ luego de una carga de $75 \mathrm{~g}$ de glucosa oral. Existe también un estado de traslape de personas que pueden compartir ambas características, las cuales tienen un riesgo doble de desarrollar diabetes que quienes únicamente tienen un estado aislado.

Guatemala es un país donde la población está en riesgo de enfermar de diabetes: solo por el hecho de ser hispanos se cree que aumenta el riesgo de padecer diabetes. En Estados Unidos los niños y niñas hispanos tiene un mayor riesgo para diabetes, en comparación con el resto de la población, en un estudio de registro la prevalencia de diabetes mellitus tipo 2 en jóvenes hispanos fue 0.79 por 1000 individuos, lo cual es 4 veces mayor que en jóvenes blancos (Dabelea et al., 2014). Además se ha demostrado que los niños hispanos tienen menor sensibilidad a la insulina (Cruz et al., 2004) y reducción de la función de las células $\beta$ pancreática (Goran et al., 2004). Por supuesto que la información anterior debe ser evaluada con cautela ya que el grupo de guatemaltecos o descendientes de guatemaltecos esta poco representado en los estudios anteriores, sin embargo, es información útil para realizar asociaciones. 
Otro factor de riesgo importante es el sobrepeso y la obesidad, según la Encuesta Mundial de Salud Escolar (MSPAS, 2009) donde se completaron 5,592 cuestionarios a estudiantes guatemaltecos entre $13 \mathrm{y}$ 15 años, el $32 \%$ de los estudiantes en establecimientos públicos estaban en sobrepeso, clasificado por índice de masa corporal y $9.2 \%$ en obesidad; comportamiento similar en establecimientos privados donde el $32 \%$ estaban en sobrepeso y $10.9 \%$ en obesidad; esto disminuye en establecimientos educativos fuera de la ciudad donde el $22.8 \%$ estaban en sobrepeso y el $5.2 \%$ en obesidad. Lo que significa que en la ciudad alrededor del $40 \%$ de los estudiantes están en sobrepeso u obesidad y en el interior aproximadamente el $28 \%$.

¿Qué se cree que pasa en la vida adulta? Se considera que aumentará el número de personas con obesidad y sobrepeso; por ejemplo, en la encuesta de adultos (a partir de los 20 años) realizada en el municipio de Villa Nueva, Guatemala en 2006, se encontró el $38.46 \%$ de personas en sobrepeso y $21.2 \%$ en algún grado de obesidad, de los 1,049 a quienes se les realizaron mediciones corporales, el $59.73 \%$ estaba en obesidad o sobrepeso (Organización Panamericana de la Salud [OPS], 2007). ¿Por qué es esto un problema? Porque ser obeso o con sobrepeso es un factor de riesgo demostrado para el desarrollo de diabetes mellitus. En un estudio realizado en los Estados Unidos se encontró que casi el $80 \%$ de los jóvenes con diabetes mellitus tipo 2 eran obesos y el 10\% con sobrepeso (Liu et al., 2010).

Retomando la Encuesta Mundial de Salud Escolar (MSPAS, 2009) existen algunos datos importantes y relevantes de mencionar, no solo para la diabetes sino para enfermedades crónicas no trasmisibles en general; se les interrogó sobre haber realizado actividad física al menos 60 min en los últimos 7 días: y el 19.2\% respondió ningún día, $27.4 \%$ un día, $14.2 \%$ dos días, lo que significa que en conjunto el $60 \%$ realiza menos de 2 días a la semana actividad física de al menos 60 min; lo recomendable para una vida saludable es por lo menos 150 min semanales de ejercicio moderado o $75 \mathrm{~min}$ de ejercicio intenso (aunque debe individualizarse). Además $28.5 \%$ de los estudiantes pasaban de 3 a $8 \mathrm{~h}$ al día sentados en actividades recreativas, es decir en sedentarismo (MSPAS, 2009). Esta encuesta es fundamental para la argumentación del autor, ya que esto demuestra que de los jóvenes guatemaltecos (futuros adultos) alrededor del $40 \%$ tienen sobrepeso $\mathrm{u}$ obesidad, $60 \%$ de ellos no hacen suficiente ejercicio y $28 \%$ vive de manera sedentaria, o sea que las piezas se están ajustando día a día para producir aún más diabéticos. ¿Se está ejecutando un plan preventivo nacional? La respuesta a esta pregunta continua siendo una interrogante; por ejemplo en el Plan de Acción 2008-2012 para la Prevención y el Control Integral de las Enfermedades Crónicas y sus Factores de Riesgo (MSPAS, 2009) se contemplan objetivos específicos para estructurar indicadores y realizar actividades puntuales según el objetivo; sin embargo, al contrastarlo con lo que sucede actualmente se puede determinar que no existen un cumplimiento de las propuestas de dicho plan de acción, al menos, no en su totalidad. Un ejemplo es que no existe una Política Nacional para Enfermedades Crónicas real en Guatemala, que según este plan iba a ser ejecutada desde el 2012.

En cuanto al tema asistencial, el MSPAS (2010) publicó las Guías para la prevención, detección, evaluación y tratamiento de las enfermedades crónicas no transmisibles, dichas guías están hechas para la atención en el primer y segundo nivel de atención, y a pesar del nombre del documento, existe tan solo una página, la número seis, dedicada a la Prevención de las Enfermedades Crónicas, donde se puntualizan las medidas a realizar, sin embargo, se aborda de manera superficial y no se detalla la manera de darle seguimiento adecuado; por ejemplo, se describen los dos tipos de pre-diabetes: (1) glicemia en ayunas alterada e (2) intolerancia a los carbohidratos; asimismo se puntualiza la manera de diagnosticar estos dos tipos de pre-diabetes, pero no se define si es adecuado o no tratar de manera farmacológica a estos pacientes o el seguimiento que deben tener; según un estudio realizado por Centros para el Control y Prevención de Enfermedades (CDC) de los Estados Unidos, revela que el tratamiento preventivo con metformina retrasa la aparición de diabetes y aún más, que este retraso es más eficaz en adultos más jóvenes de 25 a 44 años (2011).

Existen, sin embargo, algunos avances que hay que resaltar, tal es el caso de la actualización de las Guías alimentarias para Guatemala realizadas en 2012 donde se incluyen la actividad física como parte de las sugerencias rutinarias, además de promover la disminución del consumo de sal, grasas y promover el consumo de semillas (MSPAS, OPS, Instituto de Nutrición de Centro América y Panamá, 2012). Otro avance importante es la creación de la Comisión para Prevención de Enfermedades Crónicas No Transmisibles (Acuerdo Ministerial 040-2014 del MSPAS), la cual se integra por representantes de varios sectores y que podrá promover acciones eficaces para el control de este tipo de enfermedades. 
Para el verdadero control de la diabetes se debe asumir como parte de las políticas de Estado. El autor propone una estrategia preventiva; dado que la obesidad, sobrepeso y estados pre-diabéticos pueden estar presentes desde la infancia, se propone que se realice un programa nacional de tamizaje, donde exista cooperación entre el Ministerio de Educación y el MSPAS. La propuesta consiste en aprovechar la cobertura masiva a nivel de educación primaria del Ministerio de Educación, que según el INE para el 2012 la tasa neta de cobertura escolar de Guatemala era 89\% (2013b), y realizar un "reclutamiento" a quienes se detecten con factores de riesgo (obesos, sobrepeso y estados pre-diabéticos e incorporarlos a la Dirección General de Educación Física (DIGEF) y a programas de salud preventiva nutricional para el seguimiento. Además de lo anterior, se propone generar normas (incluso leyes) para que sea obligatorio el tamizaje glicémico al momento de aplicar a un trabajo público y privado, al matricularse para estudios diversificados y universidades. De esta manera el Estado tendría un primer tamizaje con niños en edad escolar y un segundo tamizaje con jóvenes-adultos que laboren o continúen sus estudios. Todos los detectados en riesgo deberán integrarse a programas de salud, nutrición (para reducción de peso) y actividades físicas amparadas por leyes que así lo detallen.

Ahora bien, en cuanto al segundo momento de la diabetes que planteó el autor-anteriormente: personas diabéticas que tendrán complicaciones relacionadas con la enfermedad, es necesario hacerse las siguientes interrogantes: ¿qué sucede en Guatemala con estos pacientes?, ¿tendrán complicaciones? o, ¿el tratamiento es adecuado para evitarlas? El lector debe comprender que el tratamiento de la diabetes es mucho más complejo que otras enfermedades; por ejemplo, si un paciente previamente sano es diagnosticado con neumonía bacteriana adquirida en la comunidad, la solución para su problema es administrar un antibiótico que tenga cobertura contra la bacteria que lo afectó, 7 a 10 días después, el paciente estará de nuevo sano. Ahora bien, en cuanto al tratamiento de la diabetes mellitus, primero se debe clasificar el tipo de diabetes que padece y luego se escoge el fármaco adecuado para cada uno; pero dado que la diabetes afecta muchos sistemas y se acompaña usualmente de otras patologías, el abordaje del paciente debe ser integral, incluyendo un tamizaje completo inicial, buscando la presencia de alguna complicación derivada de la diabetes: retinopatía, enfermedad coronaria, nefropatía, neuropatía, etcétera. Es importante la asistencia nutricional individualizada, apoyo psico- lógico, y educación en diabetes. Existen básicamente dos tipos de educación en diabetes: la tradicional y la educación en autocontrol, esta última, según estudios efectuados, ha demostrado mejorar el control de glicemia y el tratamiento adecuado de complicaciones como dislipidemia, hipertensión arterial, insuficiencia cardiaca, etcétera. Un punto importantísimo de recordar es que a diferencia del ejemplo del paciente con neumonía, el paciente con diabetes nunca se va a curar; la labor es mantenerlo libre de complicaciones y a largo plazo de muerte relacionada con la diabetes mellitus. Lo anterior nos lleva a formular dos preguntas básicas a responder: Primera ¿el tratamiento adecuado realmente es eficaz para disminuir complicaciones relacionadas con la diabetes mellitus? La respuesta es sí.

Un adecuado tratamiento reduce complicaciones, principalmente macro vasculares. Un ejemplo es un estudio danés en que se aleatorizaron 160 pacientes con diabetes mellitus 2 y microalbuminuria persistente en dos grupos; un grupo recibió terapia convencional y el otro grupo terapia intensiva, con metas de hemoglobina glicosilada $<6.5 \%$, colesterol total $<175 \mathrm{mg} / \mathrm{dL}$, triglicéridos $<150 \mathrm{mg} / \mathrm{dL}$, presión arterial sistólica $<$ $130 \mathrm{mmHg}$ y presión arterial diastólica $<80 \mathrm{mg} / \mathrm{dL}$, el objetivo principal fue medir la presencia de complicaciones micro y macro vasculares. La media de tratamiento fue de 7.8 años y se concluyó que, el tratamiento intensivo se asoció a menor riesgo de muerte por causas cardiovasculares (Hazard ratio, 0.43; 95\% intervalos de confianza, 0.19 a $0.94 ; \mathrm{p}=0.04$; esto significa una reducción del $57 \%$ de muerte) y eventos cardiovasculares (Hazard ratio, 0.41; 95\% intervalos de confianza, 0.25 a $0.67 ; \mathrm{p}=<0.01$; reducción de $59 \%$ de eventos cardiovasculares). Además un paciente en el grupo intensivo progresó a insuficiencia renal terminal comparado con seis en el grupo de la terapia convencional con un valor $\mathrm{p}=0.04$ (Gaede, Lund-Andersen, Parving, \& Pedersen, 2008).

La segunda pregunta es, ¿en Guatemala se trata adecuadamente la diabetes? La respuesta es no. Según la Iniciativa Centroamericana de Diabetes: Calidad de la Atención a la Diabetes, realizado en todos los países de Centroamérica con una muestra de 2,977 pacientes (expedientes clínicos), en los tres niveles de atención: primario, secundario y terciario (algunos países solo se evaluaron dos niveles), concluyó que, a la evaluación de información recogida en los expedientes en Guatemala no se reportaron hábitos tóxicos de los diabéticos, a pesar que es conocido que el promover la cesación del tabaquismo también reduce complicaciones relaciona- 
das con diabetes. A pesar de su importancia en la enfermedad, la medición de cintura únicamente se realizó en Costa Rica. En Guatemala el 17.7\% de los expedientes tenían registro de estatura y se pesó al $29 \%$ de los pacientes. En cuanto al examen físico general y laboratorios, en Guatemala la medición de la presión arterial se registró en $40 \%$ de los expedientes, creatinina sérica $12 \%$, colesterol $23 \%$, triglicéridos $22 \%$, hemoglobina glicosilada $12.1 \%$ y no se realizó examen de laboratorio de proteínas en orina, ni examen de la vista y de pies. Además, Guatemala no tiene registro de mensaje educativo, esto incluye alimentación, cuidado de pies, actividad física, toma de medicamentos, identificación de hipoglicemia y ajuste de dosis de insulina (Barceló et al., 2004). Estos datos nos pueden sugerir que en Guatemala no se realizó una evaluación adecuada del paciente diabético en las instituciones participantes en el estudio descrito, Hospital General San Juan de Dios, Instituto Guatemalteco de Seguridad Social y Hospital Roosevelt. En cuanto al control de diabetes (metas terapéuticas de los pacientes evaluados en el estudio antes mencionado), se encontró que las medias de glicemia en ayunas en Guatemala fue de $187.8 \mathrm{mg} / \mathrm{dL}$, glicemia al azar $247 \mathrm{mg} / \mathrm{dL}$, creatinina $1.59 \mathrm{mg} / \mathrm{dL}$, trigliceridos $265 \mathrm{mg} / \mathrm{dL}$, hemoglobina glicosilada $8.65 \%$, colesterol LDL 103 mg/dL (Barceló et al., 2004). En base a los datos mencionados, se puede inferir que los pacientes tratados están metabólicamente descontrolados, según este estudio solo el $27 \%$ de los pacientes guatemaltecos están controlados, es decir alcanzan las metas terapéuticas, lo que significa que no se está reduciendo el riesgo de complicaciones y muerte.

En cuanto a la terapéutica utilizada en Guatemala el $63 \%$ de los tratantes utilizan hipoglucemiantes orales como monoterapia, de los cuales, solo el $44 \%$ fue metformina (Barceló et al., 2004), a pesar que existe evidencia sólida que la metformina es el tratamiento farmacológico más importante de la diabetes mellitus 2, ya que reduce la hemoglobina glicosilada en aproximadamente $1.5 \%$, mejora el perfil de lípidos y disminuye ligeramente de peso (DeFronzo \& Goodman, 1995). Entonces, si analizamos la atención a los pacientes diabéticos en base al estudio mencionado, podremos asumir que al no lograr metas terapéuticas, sufrirán complicaciones y muerte prematura.

El autor está convencido que existe una solución para superar este problema. Sabiendo de antemano que más de la mitad de la población guatemalteca está en pobreza y que el MSPAS no tiene el abastecimiento ni asistencia médica adecuada hacía los pacientes y que a causa de estos dos factores el tratamiento es inadecuado, deberá de promoverse la creación de un programa nacional e integral para el tratamiento farmacológico y no farmacológico de la diabetes mellitus, el cual deberá tener como objetivo principal la creación de clínicas especializadas en diabetes erigidas en todo el país, principalmente en cabeceras departamentales o en caso de departamentos muy extensos y de difícil acceso, en municipios priorizados. Dichas clínicas deberán tener médicos con especialidad en medicina interna o diabetólogos, además médicos generales, educador en diabetes, enfermeras profesionales, nutricionistas y exámenes de laboratorio pertinentes para la enfermedad. Dichas clínicas tendrán la capacidad para: (1) atender consulta ambulatoria de pacientes, (2) entregar ( $\sin$ costo) medicamento de calidad (hipoglucemiantes orales o insulina según la requiera), (3) dar plan dietético individualizado por nutricionista, (4) dar grupo de apoyo o club de diabéticos donde se impartirá educación para el autocontrol de la diabetes, que según la FID (2011): "la educación para personas con diabetes no es una opción: es un imperativo" y (5) realizar exámenes de laboratorio que se necesitan para el control adecuado de la enfermedad.

La solidez y eficacia en cuanto al tratamiento de esta patología según el plan antes descrito, no está en cuestión, sino la factibilidad de la misma. Aunque aún se desconoce el impacto social y económico para Guatemala que podría tener la reducción de complicaciones con base a un adecuado tratamiento. Sin embargo, en Estados Unidos en 2009 un análisis del sistema de salud, mostró que el costo promedio anual para un plan de salud de un diabético con alguna complicación fue en promedio tres veces más costoso que un plan de salud de un paciente diabético sin complicaciones (Vojta, De Sa, Prospect, \& Stevens, 2012). Por lo que la idea de esta estrategia terapéutica quizá sea acertada.

El tercer momento, personas con diabetes mellitus y complicaciones a causa de la enfermedad que finalmente morirán, tiene relación estrecha con su aparecimiento, al no tener un tratamiento adecuado.

La relación entre el aparecimiento de complicaciones y la diabetes es clara, por ejemplo, según la FID (2013), en Estados Unidos se estima que los adultos con diabetes tienen tasas de mortalidad por enfermedad cardiaca 2 a 4 veces más que los adultos sin diabetes, además que el riesgo de sufrir accidentes cerebrovasculares es de 2 a 4 veces más alto en personas con diabetes. Hablando de hipertensión, para el período 2005-2008, el $67 \%$ de adultos de 20 años o más que tenían diabetes, 
tenían presión arterial mayor a 140/90 $\mathrm{mmHg}$ o tomaban antihipertensivo; además se sabe que la diabetes es la principal causa de ceguera en adultos entre los 20-74 años; un aspecto preocupante es la enfermedad renal, según estos mismos datos la diabetes fue la principal causa de insuficiencia renal, y en cuanto a amputaciones no traumáticas se estimó que el $60 \%$ de estas fueron causadas por diabetes (CDC, 2011).

\section{Conclusiones}

Luego del análisis anterior, se puede sintetizar que la incidencia de diabetes en Guatemala está en ascenso y que ocupa las primeras causas de mortalidad general en el país. Además, que Guatemala posee las condiciones para que su población se enferme de diabetes, obesidad juvenil y adulta, falta de actividad física, sedentarismo y que no se cuenta con planes claros de prevención ni detección de personas con factores de riesgo a edades tempranas. Asimismo, para quienes ya están enfermos no existen los medios del tratamiento adecuado que prevengan complicaciones médicas relacionadas con la diabetes, por lo que se estima que existirá un porcentaje alto de complicaciones. El autor propone dos soluciones posibles a esta problemática: un sistema de tamizaje en edades escolares y adultos; además de la creación de un programa nacional e integral para el tratamiento farmacológico y no farmacológico de la diabetes mellitus. Su factibilidad financiera por supuesto, es discutible; por lo que regresar al enfoque preventivo sea quizá lo más adecuado y esperar que en algunas décadas observemos un cambio. Aun así, habrá que preguntarse si el tratamiento adecuado y accesible para toda la población es menos costoso, que la carga que representan las complicaciones relacionadas con la diabetes. Desde el punto de vista productivo, la argumentación social y económica se analiza la situación de la siguiente manera: el paciente, con factores de riesgo, pero aun sano es económicamente activo es una persona que produce para el país y mueve la economía, una persona productiva trabaja para generar dinero para sí y en forma de impuestos, además consume productos o servicios de otros. La persona diabética ingresa dentro del rubro de gastos a sistemas de salud directos e indirectos, asimismo produce menos a causa de la enfermedad. Mientras que la persona con diabetes que sufre alguna complicación: ceguera, amputaciones, enfermedad crónica renal, enfermedades cardiovasculares, etcétera, se torna una persona que no es económicamente activa, sino representa un gasto para los sistemas de salud (el paciente y en algunos casos, algún familiar que no trabaja para su cuidado); y si se analiza la calidad de vida, los pacientes y su entorno familiar, se van deteriorando conforme aparecen las complicaciones.

Lo paradójico del tema es que se sabe lo que se está haciendo mal, decía Sócrates que, "la ciencia humana consiste más en destruir errores que en descubrir verdades", se posee el conocimiento y las herramientas para reorientar el camino, pero que por distintos factores, ajeno a lo puramente asistencial, no se está realizando una intervención oportuna, lo que pronostica un futuro para la diabetes, incierto y posiblemente poco alentador. Aunque así se tilde de utópico estas letras, Guatemala puede contrarrestar este y muchos de sus problemas apostándole a la voluntad de las personas, pensémonos todos, como miembros de un Estado.

\section{Referencias}

Barceló, A., Aedo, C., Rajpathak, S., \& Robles, S. (2003). The cost of diabetes in Latin America and the Caribbean. Bulletin of the World Health Organization, 81(1), 19-21.

Barceló, A., Pérez E., Cafiero, E., Meiners, M., Gregg E., Gerzoff, B., ... Pastora, M. (2004) Iniciativa Centroamericana de Diabetes: Calidad de la atención a la diabetes. Organización Panamericana de la Salud, Iniciativa Centroaméricana de Diabetes. Recuperado de http://portal.mspas.gob.gt/files/ Descargas/ProtecciondelaSalud/Bancos\%20 de\%20Sangre/iniciativa_centroamericana.pdf

Centros para el Control y la Prevención de Enfermedades. (2011). Hoja informativa nacional de diabetes: información general y cálculos nacionales sobre la diabetes y prediabetes en Estados Unidos 2011. Atlanta, GA: Departamento de Salud y Servicios Humanos de las Estados Unidos, Centros para el Control y la Prevención de Enfermedades, Centro Nacional para la Prevención de Enfermedades Crónicas y Promoción de la Salud. Recuperado de http://www.cdc.gov/diabetes/pubs/pdf/ ndfsspanish.pdf 
Cochrane Library. (2015). Prevalence. En Wiley Online Library. Recuperado de http://onlinelibrary.wiley. com/cochranelibrary/search/mesh?searchRow. searchCriteria.meshTerm=prevalence\&searchMes$\mathrm{h}=$ Lookup\&searchRow.ordinal $=0$ \&hiddenFields. strategySortBy=last-modified-date\%3Bdesc\&hiddenFields.showStrategies $=$ false\&hiddenFields. containerId $=$ \&hiddenFields.. tag $=$ \&hiddenFields . originalContainerId

Cruz, M. L., Weigenserg, M. J., Huang, T. T., Ball G., Shaibi, G. Q., \& Goran, M. I. (2004). The metabolic syndrome in overweight Hispanic youth and the role of insulin sensitivity. Jounal of Clinical Endocrinoogyl and Metabolism, 89(1), 108-113. doi: 10.1210/jc.2003-031188

Dabelea, D., Mayer-Davis, E. J., Saydah, S., Imperatore, G., Linder, B., Divers, J., ... Hamman, R. F. (2014). Prevalence of type 1 and type 2 diabetes among children and adolescents from 2001 to 2009. The Journal of the American Medical Association, 311(17), 1778-1786. doi: 10.1001/ jama.2014.3201.

DeFronzo, R. A., \& Goodman, A. M. (1995). Efficacy of metformin in patients with non-insulin-dependent diabetes mellitus. The Multicenter Metformin Study Group. New England Journal of Medicine, 333(9), 541-549.

Federación Internacional de Diabetes. (2011). Plan Mundial contra la Diabetes 2011-2021. Recuperado de http://www.idf.org/sites/default/files/ attachments/GDP-Spanish.pdf

Federación Internacional de Diabetes. (2013). Atlas de la Diabetes de la FID (6 $6^{\mathrm{a}}$ ed.). Recuperado de www.idf.org/diabetesatlas

Gaede, P., Lund-Andersen, H., Parving, H., \& Pedersen, O. (2008). Effect of a multifactorial intervention on mortality in type 2 diabetes. New England Jounal of Medicine, 358(6), 580-591. doi: 10.1056/NEJMoa0706245

Goran, M. I, Bergman, R. N, Avila, Q., Watkins M., Ball, G. D., Shaibi, G. Q., ... Cruz M. L. (2004). Impaired glucose tolerance and reduced beta-cell function in overweight Latino children with a positive family history for type 2 diabetes. Journal of Clinical Endocrinology and Metabolism. 89(1), 207-212.
Instituto Nacional de Estadística, Gobierno de Guatemala. (2013a). Caracterización estadística República de Guatemala 2012. Recuperado de http://ine. gob.gt/sistema/uploads/2014/02/26/5eTCcFlHErnaNVeUmm3iabXHaKgXtw0C.pdf

Instituto Nacional de Estadística. (2013b). Indicadores sociodemográficos. Recuperado de http://www. ine.gob.gt/index.php/estadisticas/tema-indicadores

Liu, L. L., Lawrence, J. M., Davis, C., Liese, A. D., Pettitt, D. J., Pihoker, C., ... Kahn, H. S. (2010). Prevalence of overweight and obesity in youth with diabetes in USA: the SEARCH for Diabetes in Youth study. Pediatric Diabetes, 11(1), 4-11. doi:10.1111/j.1399-5448.2009.00519.x.

Ministerio de Salud Pública y Asistencia Social. (2008). Plan de Acción 2008-2012 para la prevención y el control integral de las enfermedades crónicas y sus factores de riesgo. Recuperado de http://portal.mspas.gob.gt/files/Descargas/ProtecciondelaSalud/Bancos\%20de\%20Sangre/Plan_de_Accion.pdf

Ministerio de Salud Pública y Asistencia Social. (2009). Encuesta Mundial de Salud Escolar. Recuperado de http://portal.mspas.gob.gt/files/Descargas/ProtecciondelaSalud/Enfermedades $\% 20$ cronicas $\% 20$ no $\% 20$ transmisibles/informe_final_encuesta_escolar_julio_2011.pdf

Ministerio de Salud Pública y Asistencia Social. (2010). Guías para la prevención, detección, evaluación y tratamiento de las enfermedades crónicas no trasmisibles. Guatemala: Autor. Recuperado de http://portal.mspas.gob.gt/files/Descargas/ProtecciondelaSalud/Enfermedades $\% 20$ cronicas $\% 20$ no $\% 20$ transmisibles/guias_atencion_enfermedades_cronicas_2011.pdf

Ministerio de Salud Pública y Asistencia Social. (2012). Diagnóstico Nacional de Salud. Guatemala. Recuperado de http://sitios.usac.edu.gt/ ipn_usac/wp-content/uploads/2011/08/Diagnostico-Salud-marzo-2012.pdf

Ministerio de Salud Pública y Asistencia Social, Organización Panamericana de la Salud, Instituto de Nutrición de Centro América y Panamá. (2012). Guías Alimentarias para Guatemala. Recuperado de http://www.paho.org/gut/index. php?option=com_docman\&task=doc_download\&gid $=493 \&$ Itemid $=$ 
Nathan, D. M., Davidson, M. B., DeFronzo, R. A., Heine, R. J., Henry, R. R., Pratley, R., \& Zinman, B. (2007). Impaired fasting glucose and impaired glucose tolerance: implications for care. Diabetes Care, 30(3), 753-9.

Organización Panamericana de la Salud. (2007). Iniciativa Centroamericana de Diabetes (CAMDI): encuesta de diabetes, hipertensión y factores de riesgo de enfermedades crónicas. Villa Nueva, Guatemala, 2006. Washington, D.C.: Autor.

Real Academia Española. (2014). Prevalencia. En Diccionario de la lengua española $\left(23^{\mathrm{a}} \mathrm{ed}\right.$.). Recuperado de http://lema.rae.es/drae/?val=prevalencia

Rhys, W. (2002). Diabetes: Los costes indirectos por pérdida de productividad. Diabetes Voice, 47(3), 41-45.

Vojta, D., De Sa, J., Prospect, T., \& Stevens, S. (2012). Effective intervention for stemming the growing crisis of diabetes and prediabetes: a national payer's perspective. Health Affairs, 31(1), 20-26. doi: 10.1377/hlthaff.2011.0327

World Heart Organization. (2015). Diabetes: the cost of diabetes. En Media Centre, Fact sheet 236. Recuperado de http://www.who.int/mediacentre/ factsheets/fs236/en/ 